\title{
Designer DNA Architectures for Bionanotechnology
}

\author{
Hao Yan \\ Department of Chemistry and Biochemistry \& \\ The Biodesign Institute \\ Arizona State University \\ Tempe, AZ \\ hao.yan@asu.edu
}

The central task of nanotechnology is to control motions and organize matter with nanometer precision. To achieve this, scientists have investigated a large variety of materials including inorganic materials, organic molecules, and biological polymers as well as different methods that can be sorted into so-called "bottom-up" and "topdown" approaches. Among all of the remarkable achievements made, the success of DNA self-assembly in building programmable nanopatterns has attracted broad attention. Self-assembling DNA nanostructures assembled in this fashion can be modified in a number of ways to contain functional materials with useful biological and electronic properties. This 'bottom-up' type of approach has enormous value in the development of "molecular printboards" with resolution exceeding current nanolithographic methods. This talk will discuss some of our recent progress in using DNA as an information-coding polymer for bionanotechnology applications. Specifically, I will discuss our strategy of engineering 3D DNA origami architectures with complex curvatures and discuss perspectives of how to scale up DNA origami nano-constructions. I will use DNA directed self-assembly of hetero-elements as examples to demonstrate potential of structural DNA nanotechnology in practical applications ranging from biophysics to nano-theronostics to energy transfers. 